\title{
On the Nucleation of Ice in Highly Supersaturated Regions of Clouds
}

\author{
BRADLEY A. BAKER \\ University of Washington, Geophysics Program, Seattle, Washington*
}

16 September 1990 and 8 March 1991

\begin{abstract}
We simply but quantitatively analyze the hypothesis that the common observation, that ice crystal concentrations in clouds often far exceed measured ice nucleus concentrations, can be explained by our inability to reproduce cloud supersaturations during laboratory measurements of ice nuclei together with the fact that the probability of ice nucleation increases with supersaturation. We find the general hypothesis, while qualitatively appealing, is quantitatively unlikely. Especially unlikely is the particular hypothesis that the extremely high supersaturation regions that form around freezing drops contribute significantly to ice in clouds. The reasons for this are twofold: 1) the drop also warms the region of high supersaturation and 2) the region is very small compared to the regions of cloud with high ice concentrations.
\end{abstract}

\section{Introduction}

A major problem in cloud physics is the discrepancy between measured concentrations of ice in clouds and of ice nuclei active at cloud top temperature. There is a shortage of well-substantiated secondary ice multiplication mechanisms to explain this discrepancy (Pruppacher and Klett 1980; Jiusto and Lavoie 1975; Vali 1975; Mossop 1985). The ratio of ice crystal concentration to ice nucleus concentration, called the ice enhancement ratio, can be as large $10^{4}$ to $10^{5}$ at temperatures between $-5^{\circ}$ and $-15^{\circ} \mathrm{C}$ and decreases with decreasing cloud top temperature to unity between $-25^{\circ}$ and $-30^{\circ} \mathrm{C}$ (Pruppacher and Klett 1980).

There are a variety of ice nucleus measuring techniques and results, and there is some ambiguity as to which modes of activation are being measured by each technique. However, there is evidence that the number concentration of ice nuclei $\left(N_{i}\right)$ increases with increasing supersaturation with respect to ice $\left(S_{i}\right)$ and that for a given aerosol the following relationship approximately holds:

$$
N_{i}=C S_{i}^{\alpha}
$$

where $C$ and $\alpha$ are constants; $\alpha$ varies from 2 to 10 (28 for natural aerosol) depending on the particle type (Huffman 1973; Jiusto and Lavoie 1975; Vali 1975; Hussain and Saunders 1984). Such a relationship seems reasonable since nucleation of liquid droplets

\footnotetext{
* Present affiliation: National Center for Atmospheric Research/ MMM.
}

Corresponding author address: Dr. Bradley A. Baker, NCAR/ MMM, P.O. Box 3000, Boulder, CO 80307. follows a similar relationship. According to this relationship $N_{i}$ does depend on $T$ in the expected way since the equilibrium vapor pressure over ice decreases with decreasing temperature.

In laboratory measurements of $N_{i}$, it is difficult to achieve values of $S_{i}$ much in excess of water saturation, due to rapid condensation of liquid water. Thus, the finding that measured ice concentrations are greater than ice nucleus concentrations refers to measurements at $\left.S_{i} \approx S_{i}\right|_{\text {at water sat. In natural clouds, however, there }}$ are circumstances that can lead to supersaturations with respect to water $\left(S_{w}\right)$ considerably in excess of 0 . It has been suggested (Gagin 1972; Nix and Fukuta 1974; Gagin and Nozyce 1984; Fukuta and Lee 1986; Rangno and Hobbs 1991) that the increased ice nucleating ability of particles at high $S_{w}$ might explain ice enhancement in some clouds.

\section{Quantifying the hypothesis}

In order to quantitatively estimate the above effect on ice nucleation in clouds, let us assume (1) and define a local ice enhancement ratio $f_{l}$ to be the ratio of the number of ice nuclei activatable at the maximum cloud $S_{i}$ to the number of ice nuclei activatable at the maximum laboratory $S_{i}$ :

$$
f_{l} \equiv \frac{S_{i}^{\alpha}(\text { in cloud })}{S_{i}^{\alpha}(\text { in lab })} .
$$

Here $f_{l}$ averaged over a large volume of cloud $\left(f_{\text {ave }}\right)$ provides an estimate of the ice enhancement in that cloud due to supersaturations there being greater than achievable in the laboratory.

In order to account for typical cloud observations (Braham 1964; Auer et al. 1969; Mossop 1970; Pruppacher and Klett 1980; Rangno and Hobbs 1991) $f_{\text {ave }}$ 
should be at least $10^{3}$ at temperatures between $-5^{\circ}$ and $-15^{\circ} \mathrm{C}$. We translate (2) into the minimum cloud $S_{w}$ necessary such that $f_{l}=10^{3}$. In order to do this we must choose a maximum $S_{i}$ achieved in the laboratory, a value for $\alpha$, and a cloud temperature at which to convert the necessary cloud $S_{i}$ such that $f_{l}=10^{3}$ into the necessary $S_{w}$, such that $f_{l}=10^{3}$. We will use cloud top temperature for this since any warmer temperature would imply a higher $S_{w}$. Typically laboratory measurements of ice nuclei are done at cloud top temperature and at a maximum $S_{w}=0$. We assume this in order to determine a maximum $S_{i}$ for the laboratory and in Fig. 1 plot the cloud $S_{w}$ necessary (at cloud top temperature) such that $f_{l}=10^{3}$ versus $\alpha$ for four cloud top temperatures between $-5^{\circ}$ and $-15^{\circ} \mathrm{C}$ and for $\alpha$ between 3 and 8 .

Typical maximum $S_{w}$ in clouds are considered to be $\$ 1 \%$ (Pruppacher and Klett 1980). Thus it is questionable whether the $S_{w}$ indicated in Fig. 1 occur over large regions in any natural cloud and quite unlikely that they routinely occur over large regions of the ordinary, often mundane clouds that can exhibit ice enhancement. There are, however, small regions of cloud that do experience very high $S_{w}$.

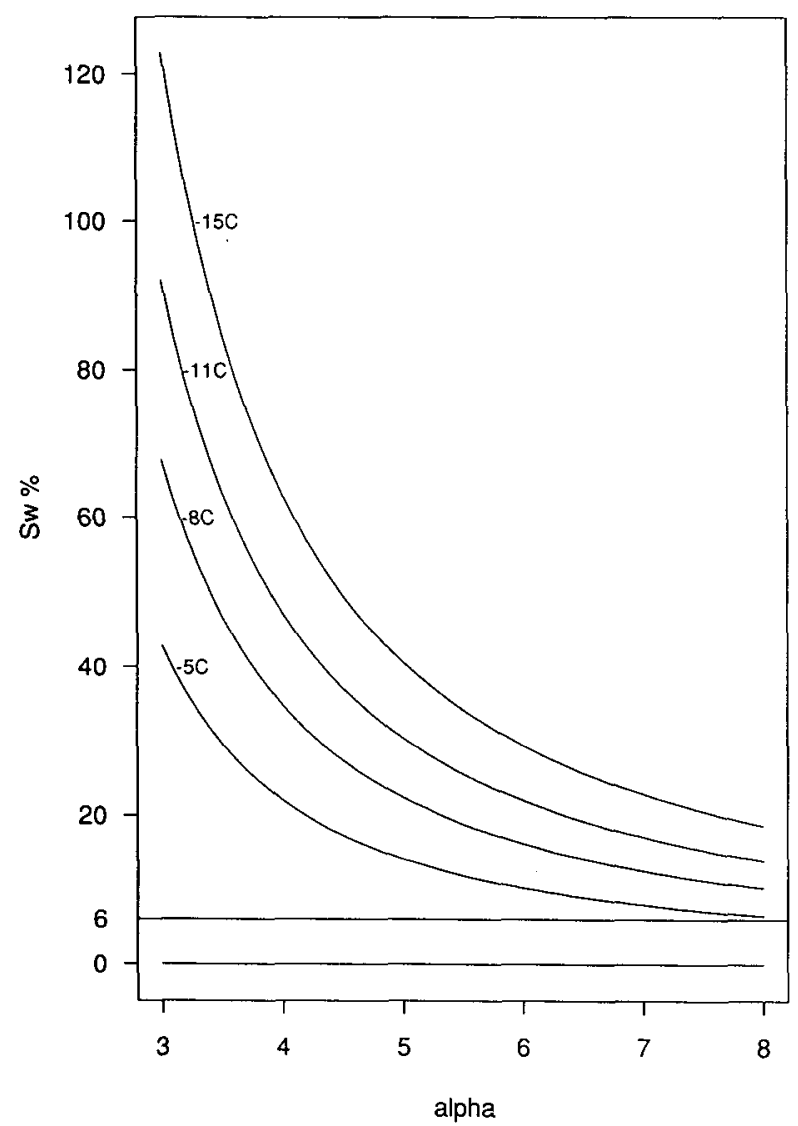

FIG. 1. The minimum cloud supersaturation with respect to water required such that $f_{l}=10^{3}$ versus $\alpha$ for various cloud top temperatures. Also drawn is the line $S_{w}=6$.

\section{Supersaturation around freezing drops}

When ice is nucleated in a supercooled drop, the drop temperature quickly rises to $0^{\circ} \mathrm{C}$. While it is freezing, the drop is a source of both heat and water vapor for the environment. The diffusivities of heat and water vapor are nearly equal, so the temperature and water vapor density profiles around the drop are similar. Since the saturated vapor density is a nonlinear function of $T$, large values of $S_{w}$ can occur around the drop. Both Dye and Hobbs (1968) and Rosinski et al. (1975) observed the formation of droplets around freezing drops and calculated the $S_{w}$ profile around a freezing drop. The latter also observed activation of ice nuclei near freezing drops. Gagin and Nozyce (1984) have indirectly observed the activation of ice nuclei around freezing drops in an experiment originally designed to study the possibility of ice splinters being ejected from freezing drops.

Since the ice nucleating ability of aerosol increases rapidly with $S_{i}$, it might be thought that the high $S_{w}$ regions around freezing drops could be of importance to ice initiation of clouds. However, because the drop also warms the air around it, the $S_{w}$ necessary for a given $f_{l}$ is higher than that indicated in Fig. 1. Also, the volumes affected by large $f_{l}$ around freezing drops are be small compared to the large volumes of clouds exhibiting ice enhancement. Therefore the effect of those regions on a cloud must be very small. In order to demonstrate this, we calculate the local ice enhancement factor [Eq. (2)] around a freezing drop. We simplify the situation by making assumptions that will overestimate the ice enhancement effect. Therefore, when the calculated effect is small, the real effect in clouds is probably even smaller.

We assume Eq. (1) and use $\alpha=8$, since this is the maximum value observed for natural aerosol. We again assume the $S_{i}$ achieved in laboratory measurements corresponds to $S_{w}=0$ at the cloud top temperature. We also assume the drop is freezing in isolation, which ignores the effect of vapor sinks (nearby droplets and activating $\mathrm{CCN}$ and, for droplets freezing onto graupel, the graupel surface). We ignore the effects of radius of curvature, solutes, ventilation and assume spherical symmetry, and we assume all ice nuclei active at a given $S_{i}$ [predicted by Eq. (1)] are activated immediately upon subjection to their critical value. Even though ice nucleation is actually a time-dependent phenomenon (Pruppacher and Klett 1980), the effects of ventilation are substantial for large drops and graupel. The volume of air affected by high supersaturation might increase due to ventilation. However, the greater the ventilation, the shorter the time an aerosol has to be affected by the high supersaturation. We believe that our other assumptions sufficiently exaggerate the possible effect of the freezing drops so we may be justified in ignoring ventilation. Likewise, ignoring radius of curvature underestimates the effect but this is negligible 
compared to the other overestimates. The diffusivities of heat and water vapor are nearly equal, therefore the differences between the time dependent ( $\mathrm{Nix}$ and $\mathrm{Fu}$ kuta 1974) and the simple steady-state solution we use are too small to be relevant in this application. We assume steady-state boundary conditions, namely, at the drop surface $T_{o}=0^{\circ} \mathrm{C}$ and $\rho_{o}=$ water saturation, and far away $\rho_{\infty}=$ water saturation at $T_{\text {cloud top }}$, and then solve the diffusion equation for the water vapor density $[\rho(r)]$ and temperature $[T(r)]$ as functions of the distance $(r)$ from the center of a freezing droplet of radius $r_{o}$ :

$$
\begin{aligned}
\rho(r)=\rho_{\infty}+\left(\rho_{o}-\rho_{\infty}\right) \frac{r_{o}}{r}, & \\
T(r) & =T_{\infty}+\left(T_{o}-T_{\infty}\right) \frac{r_{o}}{r} .
\end{aligned}
$$

The supersaturations $S_{w}\left(r / r_{o}\right)$ and $S_{i}\left(r / r_{o}\right)$ are, by definition,

$$
S_{w, i}\left(r / r_{o}\right)=100\left[\frac{\rho\left(r / r_{o}\right)}{\rho_{\mathrm{sat}_{w, i}}\left(T\left(r / r_{o}\right)\right)}-1\right]
$$

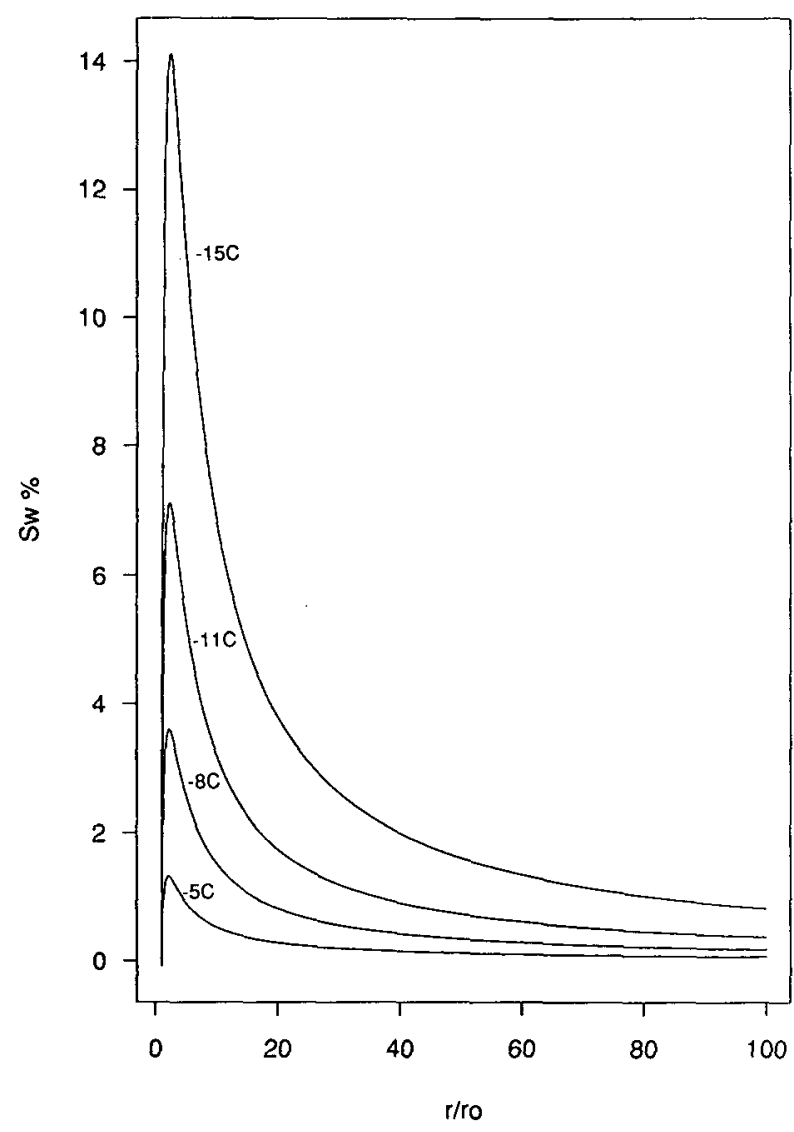

FIG. 2. Supersaturation with respect to water versus distance from the center of a drop measured in drop radii.

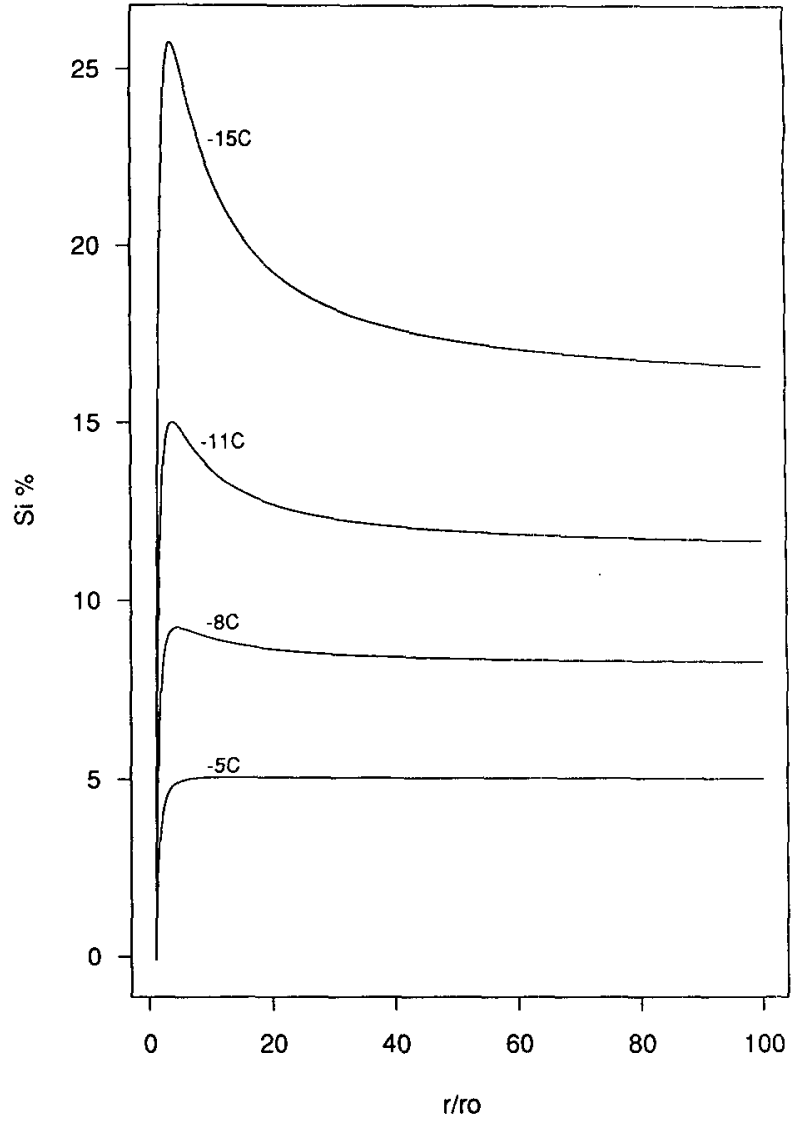

FIG. 3. As in Fig. 2 but with respect to ice.

where $\rho_{\text {sat }}(T)$ is the saturation value (with respect to water or ice) of water vapor density at temperature $T$. is

The local ice enhancement factor $\left(f_{l}\right)$ at each point

$$
f_{l}\left(r / r_{o}\right)=\frac{S_{i}^{\alpha}\left(r / r_{o}\right)}{\left.S_{i}^{\alpha}\right|_{S_{w}=0, T_{\text {cloud top }}}}
$$

Figures 2, 3, and 4, show the calculated $S_{w}\left(r / r_{o}\right), S_{i}(r)$ $\left.r_{o}\right)$, and $f_{l}\left(r / r_{o}\right)$ around a drop at four different temperatures between $-5^{\circ}$ and $-15^{\circ} \mathrm{C}$. Note that $S_{w}, S_{i}$, $f_{l}$, and the volume effected all increase with decreasing temperature.

Again in order to explain observations, $f_{\text {ave }}$ would have to be at least $10^{3}$. Since after its maximum $f_{l}$ decreases with $r$, even if we grossly underestimated the volume effected by large $f_{l}$ around a freezing drop (by ignoring ventilation), the maximum $f_{l}$ would still need to be considerably greater than $10^{3}$. However the maximum value of $f_{l}$ is less than 10 at $-11^{\circ} \mathrm{C}$ and is still less than $10^{2}$ at $-15^{\circ} \mathrm{C}$. Thus the effect that the induced high $S_{w}$ regions around freezing drops or droplets could have on ice nucleation in clouds is found to be much too small to support the proposed hypothesis. 


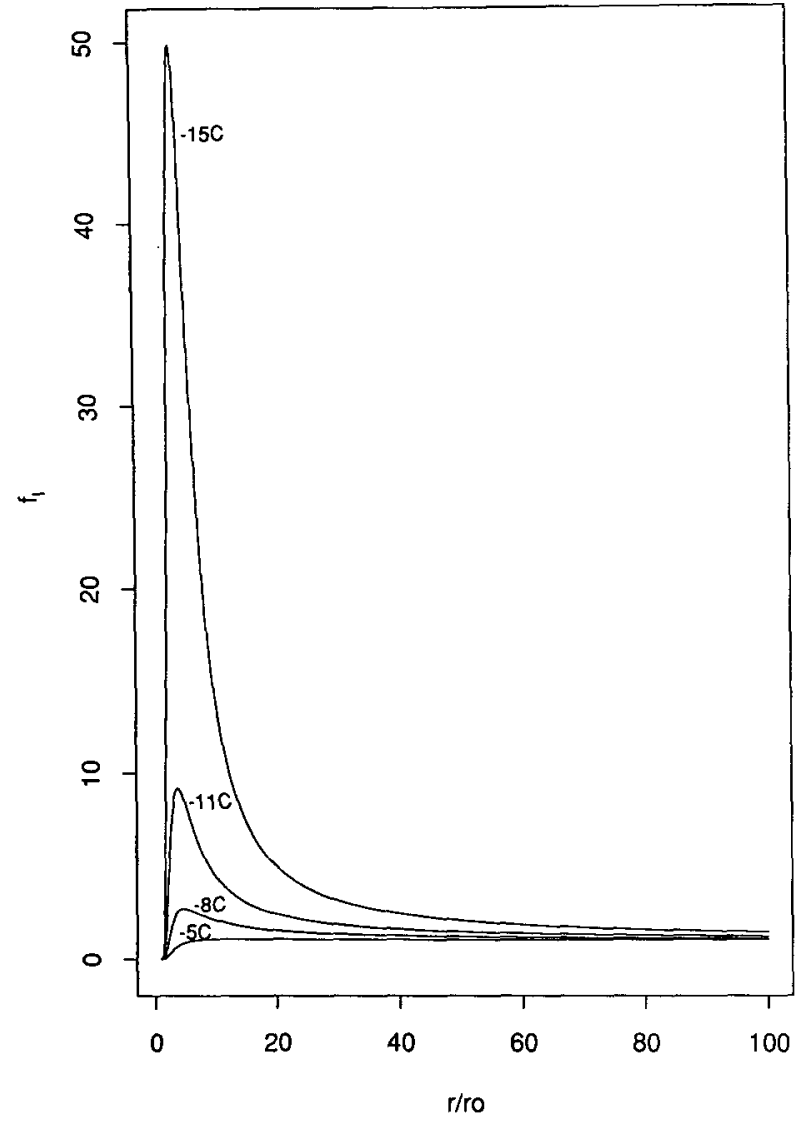

FIG. 4. Local ice enhancement factor versus distance from the center of a drop measured in drop radii.

\section{Conclusions}

We have used a simple quantitative analysis to show that the effect of the high supersaturation regions which exist in the vicinity of freezing drops is much too small to explain the discrepancy between ice crystal concentrations and ice nucleus concentrations observed in many clouds. Furthermore, we consider it unlikely that supersaturations of the magnitude necessary (Fig. 1) to explain that same discrepancy exist over large volumes elsewhere in most clouds that exhibit ice enhancement.

Acknowledgments. The author is grateful to Marcia Baker and Art Rangno for useful discussions and for help with the preparation of this manuscript. This work was supported by NSF Grant ATM-8810650.

\section{REFERENCES}

Auer, A. H., D. L. Veal and J. D. Marwitz, 1969: Observations of ice crystal and ice nuclei concentrations in stable cap clouds. $J$. Atmos. Sci., 26, 1342-1343.

Braham, R. R., 1964: What is the role of ice in summer rain showers? J. Atmos. Sci., 21, 640-645.

Dye, J. E., and P. V. Hobbs, 1968: The influence of environmental parameters on the freezing and fragmentation of suspended water drops. J. Atmos. Sci., 25, 82-96.

Fukuta, N., and H. J. Lee, 1986: A numerical study of the supersaturation field around growing graupel. J. Atmos. Sci., 43, 18331843.

Gagin, A., 1972: The effect of supersaturation on the ice crystal production by natural aerosols. J. Rech. Atmos., 6, 175-185.

- and H. Nozyce, 1984: The nucleation of ice crystals during the freezing of large supercooled drops. J. Rech. Atmos., 18, 119-129.

Huffman, P. J., 1973: Supersaturation spectra of AgI and natural ice nuclei. J. Appl. Meteor., 13, 1080-1082.

Hussain, K., and C. P. R. Saunders, 1984: Ice nucleus measurement with a continuous flow chamber. Quart. J. Roy. Meteor. Soc., 110, 75-84.

Jiusto, J. E., and R. L. Lavoie, 1975: Symposium summary. Bull. Amer. Meteor. Soc., 56, 1175-1179.

Mossop, S. C., 1970: Concentrations of ice crystals in clouds. Bull. Amer. Meteor. Soc., 51, 474-479.

- 1985: The origin and concentration of ice crystals in clouds. Bull. Amer. Meteor. Soc., 66, 264-273.

Nix, N., and N. Fukuta, 1974: Nonsteady-state kinetics of droplet growth in cloud physics. J. Atmos. Sci., 31, 1334-1343.

Pruppacher, H. R., and J. D. Klett, 1980: Microphysics of Clouds and Precipitation. D. Reidel.

Rangno, A., and P. V. Hobbs, 1991: Ice particle concentrations and precipitation development in small polar maritime cumuliform clouds. Quart. J. Roy. Meteor. Soc., 117, 207-241.

Rosinski, J., C. T. Nagamoto and T. C. Kerrigan, 1975: Heterogeneous nucleation of water and ice in the transient supersaturation field surrounding a freezing drop. J. Rech. Atmos., 9, 107-117.

Vali, G., 1975: Workshop summary. Bull. Amer. Meteor. Soc., 56, $1180-1184$. 